\title{
4. THE FUTURE
}

Probably computer links will have a major influence on all museums in the near future. Links already exist between all major centers. They exchange expertise of all sorts: security, curator experiences and images and labels of many exhibits. Several museums would benefit from the participation of professional astronomers in their design, updating and elaboration of didactic material.

Fierro, J. 1992, Teaching of Astronomy in Asian-Pacific Region, Bull. 5, Ed. Isobe Y., p. 25.

Fierro, J. and Doddoli, C. 1993, Museum News, p. 35.

Fierro, J. 1994, Teaching of Astronomy in Asian-Pacific Region, Bull. 6, Ed. Isobe Y., p. 1.

Pasachoff, J.M. and Percy, J.R. Ed. 1990, The Teaching of Astronomy, IAU Colloquium 105.

\section{THE ROLE OF AMATEURS}

Syuzo Isobe

National Astronomical Observatory, Mitaka, Tokyo 181, Japan

E-mail: oisobex@c1.mtk.nao.ac.jp

1. How to Popularize Astronomy. There are different ways to popularize astronomy. In recent years, professional astronomers have written many popular astronomy books, but only a small fraction of people, that is, less than $1 \%$ of the total population try to read them. Probably three of the most efficient ways are 1) school education, 2) social education, and 3 ) contributions of amateurs.

All people pass through a school period when some parts of astronomy are taught to school pupils. This is a great opportunity for the people to study astronomy. However, in Japan a large fraction of teachers, especially in elementary schools, did not take astronomy courses at university and therefore they cannot make their lectures attractive for school pupils. Although many pupils at the 1st or 2 nd grade of elementary schools are interested in black holes, star formation, and so on, their interest disappears after unattractive lectures. There are some efforts to escape from these difficulties, but it takes long years.

In the 1970's, many planetariums were built and those number over 400 in Japan (Isobe 1991). Then, in the period from 1984 to the present, many public observatories having a telescope with an aperture larger than $50 \mathrm{~cm}$ have been built (Isobe 1994) and those number over 50 and still increasing. This is a fortunate situation to popularize astronomy. However, nearly all the local governments who built those facilities employ only one or a few staff members who have not enough astronomical knowledge. Therefore, their activities strongly rely on the assistance of amateur 
astronomers.

Now, we can say in Japan that the contribution of amateurs is one of the most important ways to popularize astronomy.

2. Definition of Amateurs. What are amateurs? There may be different definitions. Here, we say that amateurs have a right to enjoy astronomy but also to stop doing it and that they have no obligation to popularize astronomy. Intensive efforts to do so should be done by professional astronomers, because at least they need strong public support to proceed with their astronomy.

In Japan, there are many amateurs who have produced astronomically meaningful data. The number of them is now several hundreds, although its number was 100 in Isobe's (1991) figure 1. We have many amateur groups or societies. Those are groups interested in: 1) Occultations ( $\sim 100$ members), 2) Variable stars ( $\sim 30$ members), 3 ) Comets ( 200 members), 4) Asteroids ( 200 members), 5) Moon and planets ( $\sim 50$ members), 6) Meteors ( $\sim 50$ members), 7$)$ Sun ( $\sim 30$ members), 8 ) Solar eclipses ( $\sim 30$ members), 9) Artificial satellites ( $\sim 10$ members), and 10) Novae and supernovae ( 100 members). They are making excellent observations. For example, their light curves of different variable stars are often referred to in papers of professional astronomers. In addition, over 2,000 amateurs try to take training in astronomical observations from leading amateur astronomers.

An increase in active amateurs is very helpful for professional astronomers. However, if we consider popularization of astronomy to the public ( $10^{8}$ in number), several thousand active amateurs are not enough to make a global popularization of astronomy.

3. Motivation of Amateur Activity. Most of the school teachers being active in teaching of astronomy, the staffs of planetariums and public observatories, and also the amateurs doing volunteer activity in popularization of astronomy frequently say "Let's watch stars because they are beautiful". However, this statement can easily be changed to "Lets watch flowers because they are beautiful". What one likes to watch is strongly dependent on his or her hobby. There is no superiority in stars.

A motivation of active amateurs to observe different celestial objects, as shown in section 2 , is mainly to enjoy the fact that their results contribute to astronomy. Probably, their interests are just on their own objects, but not on the other objects, and astronomy as a whole.

It is clear that astronomy is an important science for humans. For example, astronomy showed that humans cannot be alive forever because of different astronomical events. A view of the universe of individual human beings is formed by watching the night sky, and by understanding the history of our universe. Then, one can realize how important it is to popularize astronomy.

4. Conclusion. Since the total population to be taught the importance of astronomy is very large $\left(10^{8}\right.$ in Japan), supporting efforts of amateurs are essential. To make popularization processes effective, it is very important to teach active amateurs the importance of astronomy as shown in section 3 . Then, we can expect that the number of people interested in astronomy will expand.

Isobe, S. 1991, Proceedings of Astron. Soc. Australia, 9, 72-75.

Isobe, S. 1994, IAU Colloquium No. 148, held in Bandung, in press. 